\title{
Clinical Course of Relapsing Remitting Multiple Sclerosis Post-Natalizumab
}

\author{
Maximillian D. J. Fiander, Virender Bhan, Samuel A. Stewart, Natalie E. Parks
}

\begin{abstract}
Natalizumab is an efficacious disease modifying therapy (DMT) for relapsing remitting multiple sclerosis (RRMS), often limited by risk of progressive multifocal leukoencephalopathy. We describe the clinical course of RRMS patients switched from natalizumab to another DMT. We identified all RRMS patients treated with natalizumab $\geq 3$ months with JC virus antibody positivity who switched to another DMT. Overall, 84 individuals switched DMT with 57 (68\%) beginning fingolimod. On fingolimod, survival without a relapse was $74 \%(55.8-85.6 \%)$ at 36 months and survival without disease progression was $78 \%(62.6-87.6 \%)$ at 36 months. In conclusion, fingolimod is an effective therapy post-natalizumab.

RÉSUMÉ: L'évolution clinique de patients atteints de la forme cyclique de la sclérose en plaques ayant opté pour un traitement autre que celui au natalizumab. Le natalizumab est un médicament modificateur de l'évolution de la sclérose en plaques (MMSP) efficace pour le traitement de la sclérose en plaques récurrente-rémittente (SEP-RR), souvent limité par le risque de la leucoencéphalopathie multifocale progressive. Nous décrivons l'évolution clinique des patients atteints de SEP-RR qui sont passés du natalizumab à un autre MMSP. Nous avons identifié tous les patients atteints de SEP-RR ayant été traités avec le natalizumab $\geq 3$ mois avec la positivité des anticorps anti-virus JC et ayant opté pour un autre MMSP. Globalement, 84 personnes ont changé de MMSP avec 57 (68\%) ayant changé au fingolimod. Parmi les patients sous le fingolimod, la survie sans rechute était de $74 \%$ $(55,8$ à $85,6 \%)$ à 36 mois et la survie sans progression était de $78 \%(62,6$ à 87,6\%) à 36 mois. En conclusion, le fingolimod est une thérapie postnatalizumab efficace.
\end{abstract}

Keywords: Disease modifying therapy, Fingolimod, Natalizumab, Multiple sclerosis, Survival analysis doi:10.1017/cjn.2019.42

Can J Neurol Sci. 2019; 46: 455-458

Relapsing remitting multiple sclerosis (RRMS) is an immunemediated demyelinating disease of the central nervous system. Disease modifying therapy (DMT) modulates or suppresses the immune system to reduce clinical relapses and new inflammatory lesions on imaging. Natalizumab is among the most effective DMT demonstrating 68\% annualized relapse rate (ARR) reduction compared to placebo. ${ }^{1}$ This humanized antibody blocks alpha-4 integrin, a protein on the surface of lymphocytes, to prevent lymphocyte migration across the blood brain barrier. The risk profile of natalizumab includes progressive multifocal leukoencephalopathy (PML) which is a potentially fatal viral brain infection. Risk of natalizumab-associated PML increases with increasing the duration of therapy among serum JC virus antibody-positive individuals. ${ }^{2}$ As such, JC virus antibody positivity limits duration of treatment with natalizumab. Among RRMS patients discontinuing natalizumab, there is a risk of rebound inflammatory activity even when initiating another DMT. ${ }^{3}$ DMT options have evolved over time with injectable (interferon- $\beta$, glatiramer acetate), oral (fingolimod, dimethyl fumarate, teriflunomide, cladribine), and additional infusion (alemtuzumab, ocrelizumab) therapies. The purpose of this study is to describe the clinical course of RRMS patients switched from natalizumab to another DMT in a Canadian MS clinic.

We conducted a retrospective study using prospectively collected data from the Dalhousie Multiple Sclerosis Research Unit (DMSRU) database. Approval was given by the Nova Scotia Health Authority (NSHA) research ethics board (REB\#:1023305).
The DMSRU database has been prospectively maintained since 1980 in Halifax, Nova Scotia, Canada. All individuals provided consent for use of health information for research. The DMSRU database was searched to identify all RRMS patients treated with natalizumab $\geq 3$ months who discontinued therapy with serum JC virus antibody-positive status and switched to another DMT (Figure 1). Data extracted from the DMSRU database included demographic information (age at RRMS diagnosis, sex), details of RRMS treatment (DMT type, DMT duration, serum JC virus antibody status), and RRMS clinical outcomes (relapses, progression). Relapses were defined as new/worsening neurologic symptoms persisting $\geq 24$ hours in the absence of fever or infection. ${ }^{4}$ Progression was assessed using Expanded Disability Status Scale (EDSS) recorded during clinical assessments performed as part of routine care. Progression was defined as EDSS increase confirmed with two assessments separated by $\geq 3$ months of $\geq 1$ point for EDSS $1.0-5.0$ and $\geq 0.5$ if EDSS $\geq 5.5$. The primary outcomes of interest were survival relapse-free and survival progression-free on subsequent DMT following natalizumab assessed using Kaplan-Meier survival analysis.

From the Division of Neurology, Dalhousie University, Halifax, Canada (MDJF, VB, NEP); Division of Neurology, University of British Columbia, Vancouver, Canada (VB); Department of Community Health and Epidemiology, Dalhousie University, Halifax, Canada (SAS, NEP)

Received October 25, 2018. Final Revisions Submitted March 4, 2019. Date of Acceptance March 27, 2019.

Correspondence to: Dr. Natalie Parks, Halifax Infirmary, Division of Neurology, Rm 3832, 1796 Summer Street, Halifax, NS, Canada. Email: nparks@ dal.ca 


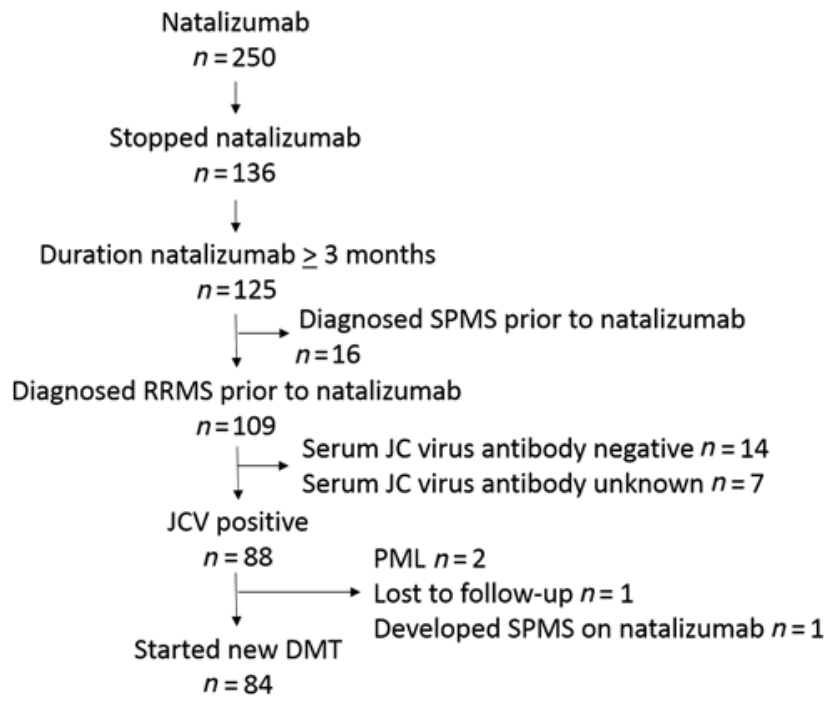

Figure 1: Consort diagram.

\section{Table 1: Baseline characteristics}

\begin{tabular}{l|l}
\hline Baseline characteristics & $n=84$ \\
\hline Sex, female:male & $59: 25$ \\
\hline Age RRMS diagnosis, median (IQR), year & $32(27-41)$ \\
\hline DMT pre-natalizumab, $n(\%)$ & $67(80 \%)$ \\
\hline Age at natalizumab initiation, median (IQR), years & $41(33-47)$ \\
\hline Duration RRMS at natalizumab initiation, median (IQR), years & $5(2-10)$ \\
\hline Duration natalizumab, median (IQR), months & $28(17-50)$ \\
\hline Washout post-natalizumab, median (IQR), months & $1(0-1)$ \\
\hline Relapse during washout, $n(\%)$ & $2(2 \%)$ \\
\hline EDSS natalizumab initiation, median (IQR) & $3.5(2.5-4.0)$ \\
\hline EDSS natalizumab discontinuation, median (IQR) & $3.5(2.0-5.0)$ \\
\hline DMT post-natalizumab, $n(\%)$ & \\
\hline Fingolimod & $57(68 \%)$ \\
\hline Dimethyl fumarate & $8(10 \%)$ \\
\hline Teriflunomide & $3(4 \%)$ \\
\hline Injectable (interferon- $\beta$, glatiramer acetate) & $5(6 \%)$ \\
\hline Alemtuzumab & $5(6 \%)$ \\
\hline Ocrelizumab & $6(7 \%)$ \\
\hline Stopped DMT post-natalizumab, $n(\%)$ & $31(37 \%)$ \\
\hline DMT &
\end{tabular}

DMT $=$ Disease modifying therapy; EDSS $=$ Expanded disability status scale; IQR = Interquartile range; RRMS = Relapsing remitting multiple sclerosis.

There were 84 RRMS patients who switched from natalizumab to another DMT that met study inclusion criteria (Table 1). The population consisted of patients with median RRMS duration of 5 years (interquartile range [IQR] $2-10$ years) where $80 \%$ had received DMT prior to natalizumab. Median duration of natalizumab treatment was 28 months (IQR 17-50 months). Overall, the washout period between switching from natalizumab to another DMT was brief with median duration 1 month (IQR

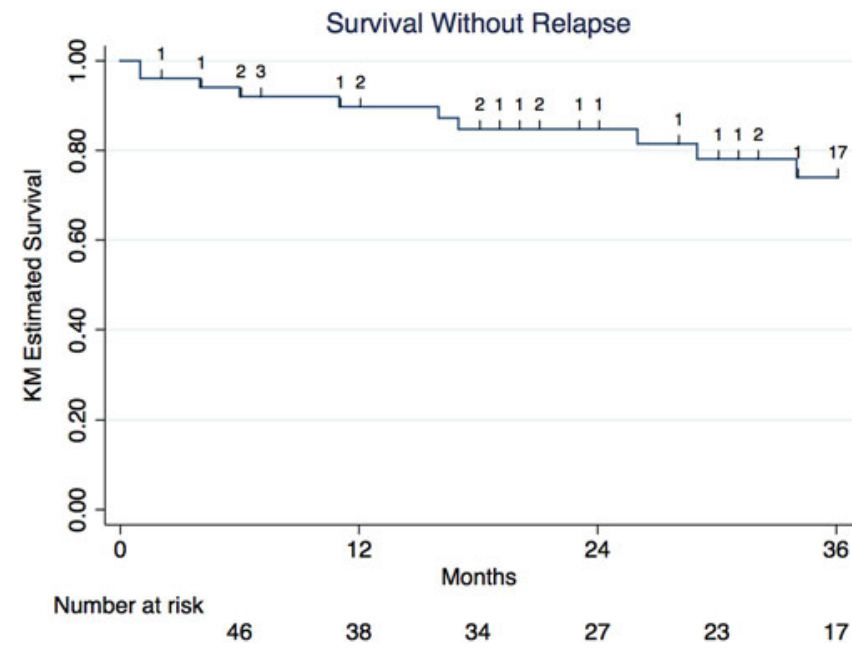

Figure 2: Kaplan-Meier survival analysis of survival relapse-free among RRMS patients treated with fingolimod post-natalizumab.

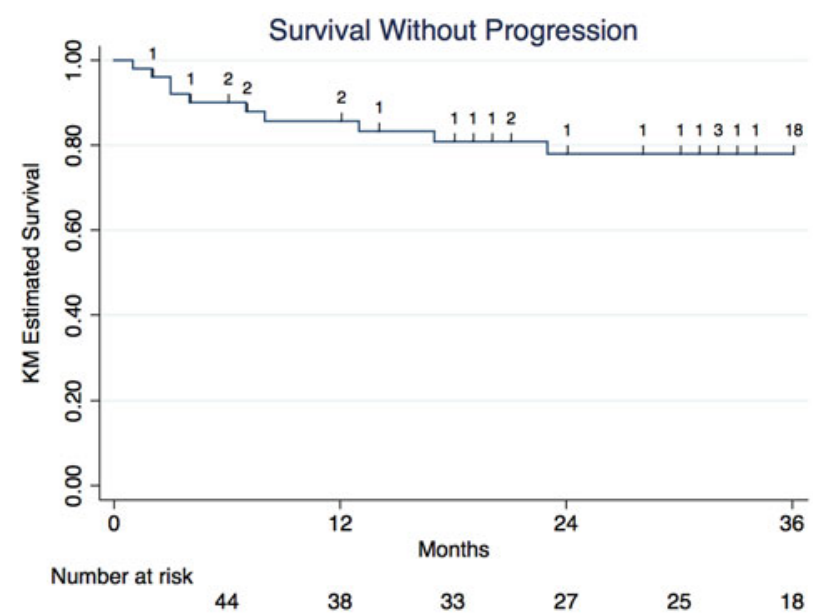

Figure 3: Kaplan-Meier survival analysis of survival progression-free among RRMS patients treated with fingolimod post-natalizumab.

0-1 months). During the washout period, two individuals experienced a relapse. Relapses occurred at 4 and 5 months, respectively, after discontinuing natalizumab but before another DMT was initiated. A majority of patients switched to fingolimod $(n=57,68 \%)$ with small numbers switching to a variety of other DMTs. Given that the majority of patients switched to fingolimod, we focused survival analysis on individuals switching to fingolimod.

Among 57 individuals switching to fingolimod, 12 individuals experienced a relapse and 12 individuals experienced disease progression after switching to fingolimod over a median treatment period on fingolimod of 31 months (IQR 12-40 months). Survival relapse-free on fingolimod was $92 \%$ (95\% confidence interval [CI] 80.1-96.9\%) at 6 months, $90 \%(77.0-95.6 \%)$ at 12 months, $85 \%$ (70.5-92.5\%) at 24 months, and 74\% (55.8-85.6\%) at 36 months (Figure 2). Survival progression-free on fingolimod was $90 \%(95 \%$ CI $77.8-95.7 \%)$ at 6 months, $86 \%(72.2-92.9 \%)$ at 12 months, $78 \%(62.6-87.6 \%)$ at 24 months, and $78 \%$ $(62.6-87.6 \%)$ at 36 months (Figure 3 ). 
Overall, we found that among RRMS patients switched to fingolimod following natalizumab, $74 \%$ remain free of relapses and $78 \%$ remain free of disease progression at 3 years. The current study found a low occurrence of relapses after switching from natalizumab to fingolimod similar to a study examining relapse rate among 89 patients switching from natalizumab to fingolimod with median washout duration of 79 days (IQR 57-96 days) after last natalizumab infusion. ${ }^{6}$ Jokubaitis et al. employed data from an international registry to calculate quarterly relapse rate on natalizumab and demonstrated that relapse rate remained relatively stable in the initial 9 months after switching to fingolimod. Relapse rate among individuals switching from natalizumab to fingolimod ranged from 0.045 to 0.11 per quarter in the 15 months prior to starting fingolimod compared to $0.079-0.13$ per quarter in the 9 months after initiating fingolimod. In addition, Jokubaitis et al. determined ARR 1.54 prior to starting natalizumab, 0.26 during natalizumab treatment, and 0.38 during fingolimod treatment. Overall, risk of relapse increased on fingolimod compared to natalizumab according to the ARR (incidence rate ratio $1.84,95 \% \mathrm{CI} 1.25-2.70$ ) but remained far less than the ARR prior to natalizumab. There was no correlation between ARR prior to natalizumab treatment and ARR during fingolimod treatment. Among those who experienced a relapse in the preceding 6 months on natalizumab, 30\% experienced a relapse during the initial 6 months on fingolimod compared to $11 \%$ experiencing a relapse during the initial 6 months on fingolimod without a relapse during the preceding 6 months of natalizumab therapy. Our study demonstrates a high portion of patients remaining free of relapses or confirmed disability progression over a longer median observation period on fingolimod of 31 months (range 0-52 months) compared to 10 months (range 3-114 months).

It is well known that there is a risk of rebound activity with a longer washout period between stopping natalizumab and starting another DMT. In Jokubaitis et al. ${ }^{6}$, a washout period of $\geq 2$ months between natalizumab and fingolimod was associated with an increased risk of relapse even after starting fingolimod. Among 333 patients switching from natalizumab to fingolimod in France, risk of relapse during the washout period was higher with increased washout duration and may affect the risk of relapse within 6 months of starting fingolimod. ${ }^{7}$ Relapses occurred among $19.9 \%$ with washout duration $<3$ months, $31.3 \%$ with washout of 3-6 months, and $59.1 \%$ with washout $>6$ months. In Cohen et al. ${ }^{7}$, washout duration was prolonged with a mean washout period of 17 weeks and only $31 \%$ of patients having a washout period $<3$ months. In the current study, the washout period was short with a median duration of 1 month reflecting current practice to transition to fingolimod in 4-6 weeks from the last natalizumab infusion.

In our clinical experience, fingolimod is the most common agent post-natalizumab with $68 \%$ of patients transitioning to another DMT receiving fingolimod. In our cohort transitioning from natalizumab to another DMT, the second most common switch was to dimethyl fumarate $(10 \%)$ while the third most common was ocrelizumab (7\%). Among a cohort of 95 Italian patients starting DMT post-natalizumab, a similar pattern was observed with 57 patients $(60 \%)$ switching to fingolimod and 7 patients (7\%) switching to rituximab. ${ }^{8}$ In Lo Re et al. ${ }^{8}, 17.5 \%$ of patients switching to fingolimod experienced clinical reactivation defined as a relapse or progression measured using EDSS, while no patient switching to rituximab experienced clinical reactivation. Among 256 Swedish patients switching from natalizumab due to JC virus antibody positivity, 142 started fingolimod and 114 started rituximab. ${ }^{9}$ Median washout period was 2.12 months for fingolimod and 1.45 months for rituximab. During the first 1.5 years of treatment, 25 (18\%) patients receiving fingolimod and $2(2 \%)$ patients receiving rituximab experienced a relapse resulting in a significantly lower risk of relapse on rituximab compared to fingolimod (hazard ratio $0.10 ; 95 \%$ CI 0.02-0.43). Rituximab has been used off-label for multiple sclerosis; however, use is often limited due to funding restrictions in Canada. Ocrelizumab, a monoclonal anti-CD20 molecule similar to rituximab, was approved in the past year by Health Canada for RRMS. In the phase III trials, ocrelizumab demonstrated $46-47 \%$ ARR reduction compared to interferon- $\beta{ }^{10}$ Although $7 \%$ of patients in our cohort switched from natalizumab to ocrelizumab, median follow-up was 0 months (range $0-5$ months) limiting our ability to assess efficacy of ocrelizumab in this cohort.

Strengths of this study include that data were obtained from a prospectively maintained database from a Canadian MS clinic. These provide real-world information concerning clinical practice for selecting DMT following natalizumab along with the efficacy of the most commonly selected DMT post-natalizumab in this cohort which was fingolimod. A weakness of this study is that it reports survival relapse-free on fingolimod following natalizumab without reporting relapse rate before or during treatment with natalizumab. Another weakness is that we did not assess radiologic disease activity.

In conclusion, fingolimod is an effective therapy following natalizumab with a high proportion of patients remaining free of relapses or progression at 3 years. Despite a variety of alternative DMTs, fingolimod remains an efficacious choice of DMT after natalizumab treatment. As new DMTs such as ocrelizumab are approved in Canada, further studies should examine efficacy of fingolimod versus alternative therapy following natalizumab.

\section{FUNDing}

There was no targeted funding for this research project. This project was made possible through the support of the DMSRU.

\section{ACKNOWLEDGMENTS}

The authors thank Dadel Gayala and Michèle Poirier for assistance in translating the abstract into French.

\section{Disclosures}

MDJF and SAS report no disclosures. VB has received honoraria for speaking, consulting, and advisory board participation from Biogen, Celgene, EMD Serono, Genzyme, Novartis, Roche, Sanofi, and Teva Neuroscience. He has acted as site principal investigator for clinical trials for Biogen Idec, EMD Serono, Novartis, Sanofi-Aventis, and Teva Neuroscience. NEP has provided consulting services to Biogen, EMD Serono, Roche, and Sanofi Genzyme. She has acted as site sub-investigator for clinical trials for Biogen, MedDay, Sanofi Genzyme, and Roche. She is the recipient of a Killam Predoctoral Scholarship and Nova Scotia Graduate Scholarship. 


\section{Statement of Authorship}

MDJF acquired/interpreted data and drafted the manuscript. VB conceptualized the study, interpreted the data, and revised the manuscript for intellectual content. SAS interpreted/analyzed the data and revised the manuscript for intellectual content. NEP conceptualized the study, acquired/interpreted/analyzed the data, and drafted the manuscript.

\section{REFERENCES}

1. Polman $\mathrm{CH}$, O'Connor PW, Havrdova E, et al. A randomized, placebo-controlled trial of natalizumab for relapsing multiple sclerosis. N Engl J Med. 2006;354(9):899-910.

2. Sorensen PS, Bertolotto A, Edan G, et al. Risk stratification for progressive multifocal leukoencephalopathy in patients treated with natalizumab. Mult Scler. 2012;18(2):143-52.

3. Rasenack M, Derfuss T. Disease activity return after natalizumab cessation in multiple sclerosis. Expert Rev Neurother. 2016; 16(5):587-94.
4. Polman CH, Reingold SC, Banwell B, et al. Diagnostic criteria for multiple sclerosis: 2010 revisions to the McDonald criteria. Ann Neurol. 2011;69(2):292-302.

5. Giovannoni G, Tomic D, Bright JR, Havrdova E. "No evident disease activity": the use of combined assessments in the management of patients with multiple sclerosis. Mult Scler. 2017;23(9):1179-87.

6. Jokubaitis VG, Li V, Kalincik T, et al. Fingolimod after natalizumab and the risk of short-term relapse. Neurology. 2014;82(14):1204-11.

7. Cohen M, Maillart E, Tourbah A, et al. Switching from natalizumab to fingolimod in multiple sclerosis: a French prospective study. JAMA Neurol. 2014;71(4):436-41.

8. Lo Re M, Capobianco M, Ragonese P, et al. Natalizumab discontinuation and treatment strategies in patients with multiple sclerosis (MS): a retrospective study from two Italian MS Centers. Neurol Ther. 2015;4(2):147-57.

9. Alping P, Frisell T, Novakova L, et al. Rituximab versus fingolimod after natalizumab in multiple sclerosis patients. Ann Neurol. 2016;79(6):950-8.

10. Hauser SL, Bar-Or A, Comi G, et al. Ocrelizumab versus interferon beta-1a in relapsing multiple sclerosis. N Engl J Med. 2017; 376(3):221-34 